International Journal of Applied Mechanics

Vol. 12, No. 7 (2020) 2050079 18 pages)

(C) World Scientific Publishing Europe Ltd.

DOI: 10.1142/S1758825120500799

\title{
Estimation of $C^{*}$-Integral for Central Cracked Plate Under Biaxial Loading
}

\author{
Yanwei Dai*,†,\|, Fei Qin*,†, Yinghua Liu ${ }^{\ddagger}$, \\ Weizhe Feng ${ }^{\S}$ and Guian Qian $₫$ \\ *Institute of Electronics Packaging Technology and Reliability \\ College of Mechanical Engineering and \\ Applied Electronics Technology \\ Beijing University of Technology, Beijing 100124, P. R. China \\ ${ }^{\dagger}$ Beijing Key Laboratory of Advanced Manufacturing Technology \\ Beijing 100124, P. R. China \\ ${ }^{\ddagger}$ Department of Engineering Mechanics \\ AML, Tsinghua University, Beijing 100084, P. R. China \\ $\S_{\text {School of Aeronautics and Astronautics }}$ \\ Dalian University of Technology, Dalian 116024, P. R. China \\ \State Key Laboratory of Nonlinear Mechanics (LNM) \\ Institute of Mechanics Chinese Academy of Sciences \\ Beijing, P. R. China \\ "ywdai@bjut.edu.cn \\ Received 25 February 2020 \\ Revised 22 July 2020 \\ Accepted 22 July 2020 \\ Published 24 September 2020
}

\begin{abstract}
The reference stress method (RSM) is a classical method to estimate $C^{*}$-integral of creep crack. An extended reference stress method (ERSM) is given for the central cracked plate (CCP) under biaxial loading in this paper. The applicability and verification for the proposed ERSM is given. The study finds that the solutions with the proposed ERSM agree better than those of RSM under biaxial loading condition. A theoretical form to predict the relationship of $C^{*}$-integral between biaxial loading and uniaxial loading is discussed. Relation between $C^{*}$-integral and creep time under biaxial loading is validated and discussed.
\end{abstract}

Keywords: Extended reference stress method; $C^{*}$-integral; biaxial loading; reference stress method; biaxial limit load.

\section{Introduction}

Structural integrity assessments have drawn a lot of attentions for cracked components under various conditions in recent years Du et al., 2017; Dai et al., 2020a, 2019a, 2020b, 2019b; Kashkoli et al., 2018; Lv et al., 2018; Pan et al., 2017]. In

$\|_{\text {Corresponding author. }}$ 
order to reasonably evaluate the lifetime of crack contained components at elevated temperature, estimation of creep fracture parameter is a crucial issue in the investigations of this area. For creeping solids, the $J$-analogy line contour integral $C(t)$-integral is the fracture parameter which is adopted to make the prediction of crack growth rate under creep regime at high temperature Riedel, 2014; Saxena, 1998]. $C(t)$-integral becomes $C^{*}$-integral if the extensive creep is approached, and it performs path-independency when the extensive creep occurs for creep crack tip field.

Note that $C^{*}$-integral is related with the line integral around the integration contour ahead of the creep crack tip, numerical computation difficulty and high expense of calculations in estimation of $C^{*}$-integral exist for complex components and structures in practical engineering practices. Furthermore, $C^{*}$-integral is a critical parameter to estimate the growth rate of creep crack extension, which is related with lifetime prediction of the crack contained structure at elevated temperature Ainsworth and Budden, 1994. Hence, it is very crucial to estimate the $C^{*}$-integral. In order to avoid the time-consuming computation cost during the evaluation of creep crack growth, a simplified method but with good precision is indeed required for engineering assessment. Reference stress method (RSM) is one such kind of simplified method to calculate $C^{*}$-integral for creep crack Fuijoka and Ainsworth, 2013; Xuan et al., 2009]. Discussions on $C^{*}$-integral with various aspects can be found in following references Kim, 2001; Kim et al., 2002; Oh et al., 2010].

Although the estimation of $C^{*}$-integral is a topic which has been discussed with efforts of many years, there still remain some problems which are not studied indepth. For example, the quantitative study of biaxial effect on $C^{*}$-integral and engineering evaluation method to estimate $C^{*}$-integral considering biaxiality are still not investigated thoroughly as far as the authors' knowledge. The discrepancies of fracture behaviors for cracked body within various materials between uniaxial loading state and biaxial loading state are obviously, which is an important topic in fracture mechanic even for creeping solids.

Regarding to the estimation of fracture parameter considering biaxiality, some investigations on $J$-integral can provide us some enlightenments. Kim et al. [2001] proposed the Enhanced Reference Stress Method (ERSM) to evaluate the $J$-integral and crack tip displacement. Lei 2004] presented a limit load solution for computation of $J$-integral of semi-elliptical surface crack. Comparing with those investigations on estimation of $J$-integral within elastoplastic solids under biaxial loading Madia et al., 2014; Miura and Takahashi, 2010], studies on effect of biaxial loading on the $C^{*}$-integral in creeping solids are quite limited. However, one deduction is almost assured, i.e., $C^{*}$-integral is surely affected by its loading state, according to studies given by $\mathrm{Xu}$ et al. [2017] and Kobayashi et al. [2017].

The aim of this paper is to present a method to estimate $C^{*}$-integral, which can take the biaxial effect into account. Towards this aim, an extended reference stress method (ERSM) is proposed, and the $C^{*}$-integral of central cracked plate (CCP) 
under biaxial loading is selected as the analyzed case to perform those numerical computations. The validation and applicability of this method are also discussed. The specific organization of this paper is given as follows. The RSM to calculate the $C^{*}$-integral with classical definition is firstly reviewed in Sec. 2, The proposed ERSM is stated in Sec. 3. The results and discussions are shown in Sec. 4 The conclusions of this paper are drawn in Sec. 5

\section{Theoretical Framework}

\subsection{Retrospect of $R S M$}

The key step to use RSM is to compute the reference stress. Herein, the reference stress $\sigma_{\text {ref }}$ is defined as follows [Ainsworth, 1984]:

$$
\sigma_{\text {ref }}=\frac{P}{\gamma P_{L}} \sigma_{y}
$$

where $P, P_{L}, \sigma_{y}$ and $\gamma$ are the applied load, the limit load, the yielding stress and modification coefficient Kim et al., 2001], respectively. $\gamma$ is identical to 1 for simple condition. With the reference stress defined in Eq. (2.1), the reference strain $\varepsilon_{\text {ref }}$ is calculated at the given reference stress, $\sigma_{\text {ref }}$, based on the true stress-strain curve of the selected material. The following formula is obtained with the definitions of reference stress and reference strain as follows Kim and Budden, 2002]:

$$
\frac{J_{P}}{J_{e}}=\frac{E \varepsilon_{\mathrm{ref}}}{\sigma_{\mathrm{ref}}}
$$

where $J_{P}, J_{e}$ and $E$ are $J$-integral under elastoplastic condition, purely elastic condition and Young's modulus, respectively.

Some researchers [Madia et al., 2014; Miura and Takahashi, 2010] considered the biaxial effect on estimation of $J$-integral in elastoplastic materials. An ERSMconsidering the biaxiality in evaluation of $J$-integral was proposed by Miura and Takahashi [2010] as follows:

$$
\frac{J_{\text {Biaxial }}}{J_{e}}=\frac{E \varepsilon_{\text {ref }}^{\text {Biaxial }}}{\sigma_{\text {ref }}^{\text {Biaxial }}}
$$

where $E, \varepsilon_{\text {ref }}^{\text {Biaxial }}$ and $\sigma_{\text {ref }}^{\text {Biaxial }}$ are Young's modulus, reference strain and reference stress under biaxial loading condition, respectively. Herein, $J_{\text {Biaxial }}$ and $J_{e}$ are the $J$-integrals under biaxial loading state and within linearly elastic materials, respectively.

According to the relations between $J$-integral and $C(t)$-integral Assire et al., 2001], there exists the following relation for $C^{*}$-integral considering creep and $C_{\text {linear }}^{*}$-integral considering linearly elastic properties with the definition of reference stress and reference strain.

$$
\frac{C^{*}}{C_{\text {linear }}^{*}}=\frac{E \dot{\varepsilon}_{\mathrm{ref}}}{\sigma_{\mathrm{ref}}}
$$


where $C^{*}$ is the $C(t)$-integral under extensive creep and $C_{\text {linear }}^{*}$ is the $C^{*}$-integral without considering creep. $\dot{\varepsilon}_{\text {ref }}$ is the reference strain with rate form, and it can be obtained by substituting reference stress $\sigma_{\text {ref }}$ into the creep constitutive equation. The computation of reference stress is the same as that defined in Eq. (2.1). As a matter of fact, $C_{\text {linear }}^{*}$ is identical to $J_{e}$. The applicability of RSM is based on the assumption that the structure is under primary load, and secondary load is not taken into account. It should be pointed out that the formula (2.4) has not taken the biaxial effect into consideration.

\subsection{Extended RSM considering biaxial effect}

Herein, an extended biaxial reference stress for a structure under biaxial loading is defined as follows with a similar form as defined by Miura and Takahashi, 2010]

$$
\sigma_{\text {ref }}^{\text {biaxial }}=\frac{P}{P_{L}^{\text {biaxial }}} \sigma_{y}
$$

where $\sigma_{\text {ref }}^{\text {biaxial }}$ denotes the reference stress of a structure under biaxial loading, and $P_{L}^{\text {biaxial }}$ is the limit load of a structure bearing biaxial loadings. The ratio of uniaxial reference stress given by Eq. (2.1) and the improved reference stress presented by Eq. 2.5) is given as follows:

$$
\frac{\sigma_{\text {ref }}^{\text {uniaxial }}}{\sigma_{\text {ref }}^{\text {biaxial }}}=\frac{P_{L}^{\text {biaxial }}}{P_{L}^{\text {uniaxial }}}
$$

where $\sigma_{\text {ref }}^{\text {uniaxial }}$ and $P_{L}^{\text {uniaxial }}$ are the reference stress and limit load under uniaxial loading, respectively.

With Eqs. (2.4) and (2.6), the $C^{*}$-integral under biaxial loading is given by

$$
\frac{C_{\text {biaxial }}^{*}}{C_{\text {uniaxial }}^{*}}=\frac{\sigma_{\text {ref }}^{\text {uniaxial }} \dot{\varepsilon}_{\text {ref }}^{\text {biaxial }}}{\sigma_{\text {ref }}^{\text {biaxial }} \dot{\varepsilon}_{\text {ref }}^{\text {uniaxial }}}
$$

where $C_{\text {biaxial }}^{*}$ and $C_{\text {uniaxial }}^{*}$ are denoted as the $C^{*}$-integral under biaxial loading and uniaxial loading, respectively. $\dot{\varepsilon}_{\text {ref }}^{\text {biaxial }}$ and $\dot{\varepsilon}_{\text {ref }}^{\text {uniaxial }}$ are the creep strain under biaxial and uniaxial states, respectively.

For a CCP, as seen in Fig. 1 the lower bound of biaxial limit load for a long plate in $y$-direction is represented as Meek and Ainsworth, 2015.

$$
P_{L}^{\text {biaxial }}=\frac{4 \sigma_{y}}{\sqrt{3}} \min \left[\frac{1}{|\lambda|}, \frac{1-a / W}{1-\lambda(1-a / W)}\right](W-a)
$$

where $\lambda$ is the stress biaxiality which is defined by $\lambda=S_{x} / S_{y}, a$ is the crack depth and $W$ is the width of plate. $S_{x}$ and $S_{y}$ are the applied loads in horizontal direction and vertical direction, respectively. The upper bounds of biaxial limit load based on slip line theory for CCP are also given by Meek and Ainsworth 2015] which is 


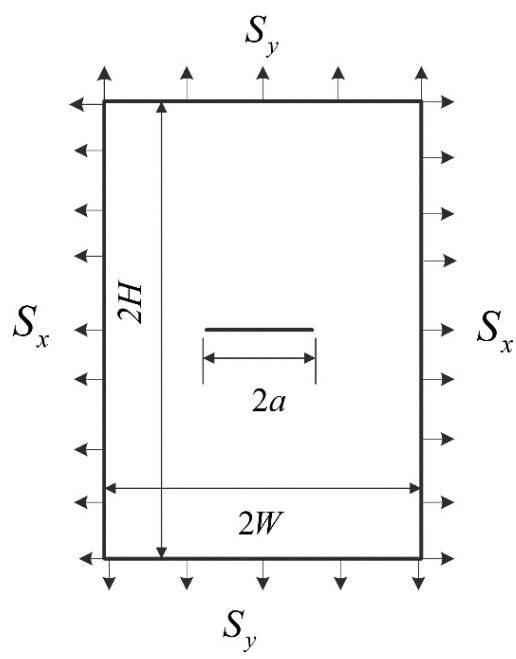

Fig. 1. CCP under biaxial loading.

given as follows:

$$
P_{L}^{\text {biaxial }}= \begin{cases}\frac{4 \sigma_{y}}{\sqrt{3}} \min \left[\frac{1-a / W}{1-\lambda(1-a / W)}\right](W-a) & \text { for }(H>W-a) \\ \frac{4 \sigma_{y}}{\sqrt{3}} \min \left[\frac{1+a / W}{1-\lambda(1-a / W)}\right](W-a) & \text { for }(H>W+a)\end{cases}
$$

where Eqs. (2.8) and (2.9) are obtained from slip line theory with two different kinds of modes, i.e., ahead of crack and behind the crack. The limit load for CCP under uniaxial loading is given as [R6, 2010]

$$
P_{L}^{\text {uniaxial }}=\frac{4 \sigma_{y}}{\sqrt{3}}(W-a)
$$

where Eq. 2.10) is available for two-dimensional CCP with thickness of $1 \mathrm{~mm}$.

With those limit load expression given in Eq. (2.9), the reference stress under biaxial loading is given as follows according to upper bound limit sloads:

$$
\begin{aligned}
& \sigma_{\text {ref }}^{\text {biaxial }}=\frac{|1-\lambda(1-a / W)|}{1-a / W} \sigma_{\text {ref }}^{\text {uniaxial }} \\
& \sigma_{\text {ref }}^{\text {biaxial }}=\frac{|\lambda(1+a / W)-1|}{1+a / W} \sigma_{\text {ref }}^{\text {uniaxial }}
\end{aligned}
$$

where those quantities in Eqs. (2.11) and 2.12 denote the same meanings as those defined in Eq. (2.8). However, for condition considering lower bound limit of Eq. (2.9), the formulae between reference stress under biaxial loading and uniaxial 
loading is given as follows:

$$
\sigma_{\text {ref }}^{\text {biaxial }}=\frac{1}{\min \left[\frac{1}{|\lambda|}, \frac{1-a / W}{1-\lambda(1-a / W)}\right]} \sigma_{\text {ref }}^{\text {uniaxial }}
$$

From Eqs. (2.8) to (2.13), the $C^{*}$-integral under biaxial loading with ERSM is presented as follows:

$$
C_{\text {biaxial }}^{*}=\frac{E \dot{\varepsilon}_{\text {riax }}^{\text {biaxial }}}{\sigma_{\text {ref }}^{\text {biaxial }}} J_{e}
$$

From Eq. (2.14), the relation between $C^{*}$-integral under biaxial loading and that under uniaxial loading is given as follows:

$$
\frac{C_{\text {biaxial }}^{*}}{C_{\text {uniaxial }}^{*}}=\left(\frac{\sigma_{\text {biaxial }}}{\sigma_{\text {uniaxial }}}\right)^{n-1}
$$

There also exists the following relations:

$$
\frac{C_{\text {biaxial }}^{*}}{C_{\text {uniaxial }}^{*}}=\left(\frac{|1-\lambda(1-a / W)|}{1-a / W}\right)^{n-1}
$$

and

$$
\frac{C_{\text {biaxial }}^{*}}{C_{\text {uniaxial }}^{*}}=\left(\frac{|\lambda(1-a / W)-1|}{1+a / W}\right)^{n-1}
$$

Herein, an analytical relation between $C^{*}$-integral under biaxial loading and $C^{*}$-integral under uniaxial loading is given. It indicates that the ratio between $C^{*}$ integral under biaxial loading and $C^{*}$-integral under uniaxial loading is dependent on creep exponent, biaxiality and crack depth ratio. In order to denote the classical RSM, the method presented in this section is denoted as ERSM. It should be emphasized that the derivations of this section does not involve any usage of material constants in detail, which indicates the solutions are theoretically applicable to all kinds of creeping materials.

\section{Material Properties and Numerical Procedures}

\subsection{Material properties}

In order to study and validate the biaxial effect on $C^{*}$-integral, the following creep constitutive equation with power-law form is adopted:

$$
\dot{\varepsilon}_{i j}=\frac{1+v}{E} \dot{S}_{i j}+\frac{1-2 v}{3 E} \dot{\sigma}_{i j} \delta_{i j}+\frac{3}{2} \dot{\varepsilon}_{0}\left(\frac{\sigma_{e}}{\sigma_{0}}\right)^{n-1} \frac{S_{i j}}{\sigma_{0}}
$$

where $\dot{\varepsilon}_{i j}, \dot{\sigma}_{i j}, \dot{S}_{i j}, \sigma_{e}, \sigma_{0}$ and $\delta_{i j}$ represent the creep strain tensor, stress tensor, deviatoric stress, von Mises equivalent stress and reference stress, respectively. The quantities with a dot form represent the differential of creep time. Herein, $n$ is the 
Table 1. Material properties used in calculations Zhao et al., 2015].

\begin{tabular}{ll}
\hline Material property & Value \\
\hline Elastic modulus $E$ & $125 \mathrm{GPa}$ \\
Poisson's ratio $v$ & 0.3 \\
Yielding stress $\sigma_{0}$ & $180 \mathrm{MPa}$ \\
Creep exponent $n$ & 5.23 \\
Creep coefficient $B$ & $2.64 \times 10^{-16} \mathrm{MPa}^{-n}$ \\
\hline
\end{tabular}

creep exponent. Creep coefficient is defined as $B=\dot{\varepsilon}_{0} / \dot{\sigma}_{0}^{n}$. For the computations, the specific material properties adopted in this paper are given in Table 1, and the data are selected from Zhao et al. [2015].

\subsection{Numerical procedures}

In fact, the contour integral implanted in ABAQUS is a robust method to calculate the $C(t)$-integral for creep crack. The $C(t)$-integral with the definition is as follows Landes and Beglev, 1976]:

$$
C(t)=\int_{\Gamma \rightarrow 0}\left[\dot{W} \mathrm{~d} y-T_{i}\left(\frac{\partial \dot{u}}{\partial x}\right) \mathrm{d} s\right]
$$

where $\dot{W}$ is the strain energy rate density, $T_{i}$ is the traction force component and $\dot{u}$ is the displacement rate. Equation (3.2) is established on the assumption that the integral contour should approach to the crack tip infinitesimally. For steady state creep, the dominant parameter $C^{*}$-integral is defined as follows Landes and Begley, 1976]:

$$
C^{*}=\int_{t \rightarrow \infty}\left[\dot{W} \mathrm{~d} y-T_{i}\left(\frac{\partial \dot{u}}{\partial x}\right) \mathrm{d} s\right]
$$

where the equation above is built upon the assumption that the creep time is very long. In calculation, the $C(t)$-integral shows the path dependence compared with $C^{*}$-integral of the path independence. To denote the transition between the $C(t)$ integral and $C^{*}$-integral, the redistribution time is always defined as

$$
t_{\text {red }}=\frac{K^{2}}{E^{\prime} C^{*}}
$$

where the determination of redistribution time $t_{\text {red }}$ is strongly dependent on the level of $C^{*}$-integral. Note that $K$ and $E^{\prime}$ are the stress intensity factor and generalized Young's modulus which depends on plain stress or plain strain states.

The finite element (FE) code ABAQUS is adopted to perform the creep analyses. A CCP is adopted to perform the numerical analysis. A one quarter model with symmetric boundary conditions is used to perform the numerical computations of CCP, and the detail model is shown in Fig. 2. The element type here is CPE4H, i.e., 
Y. Dai et al.

(a)

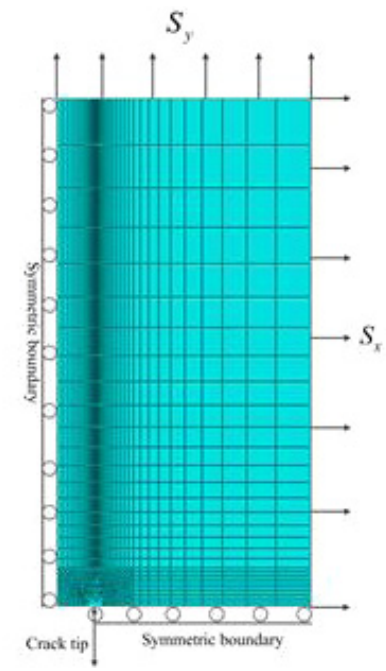

(c)

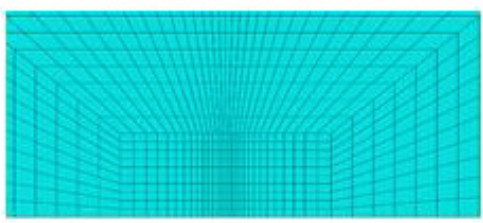

(b)

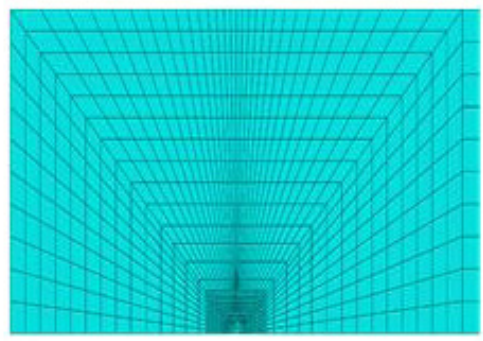

Fig. 2. FE meshes for (a) CCP, (b) crack tip region and (c) crack tip.

four nodes plane strain element with hybrid formulation. The element numbers of the computed models are between 2650 and 3560. The height and width of the CCP are $40 \mathrm{~mm}$ and $20 \mathrm{~mm}$, respectively. The strain hardening creep is used to simulate the power-law creep constitutive. The material properties can be seen in Table 1. During the analysis, the contour integral for calculation of fracture parameters, e.g., $J$-integral, stress intensity factor and $C^{*}$-integral will be used. Ten integration contours are selected to extract the computed fracture parameters, e.g., $J$-integral, stress intensity factor and $C^{*}$-integral. It should be pointed out that the computed solution of the first contour is neglected as larger numerical error could exist at the first integration contour. All the values in the following article for $J$-integral, stress intensity factor and $C^{*}$-integral are computed by averaging the computational values of the other nine contours.

\section{Results and Discussions}

\section{1. $C^{*}$-integral of CCP under biaxial loading}

In order to verify the estimation accuracy of the $C^{*}$-integral of CCP under biaxial loading with ERSM, the solutions computed with different methods are discussed in this section. Three methods are performed to make the comparisons. Those three methods are reference stress method, extended reference stress method and contour integral method calculated with ABAQUS itself, i.e., FE solutions. Herein, the RSM is the traditional RSM to compute $C^{*}$-integral. ERSM is the method proposed in Sec. 2.2 of this paper. Two different crack depth ratios are adopted to make the comparisons, i.e., $a / W=0.15$ and $a / W=0.5$. 


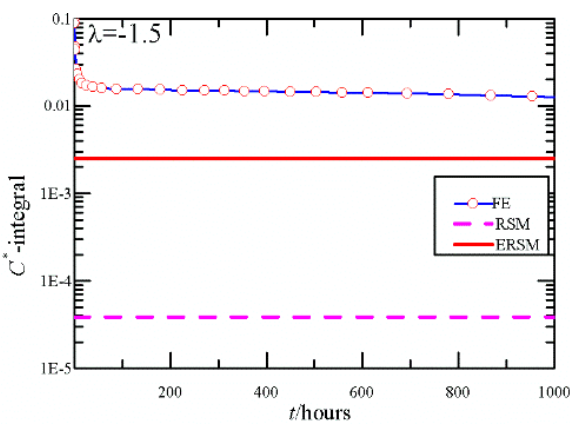

(a)

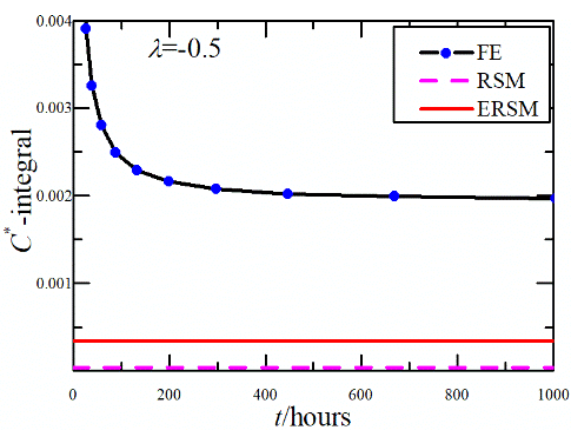

(c)

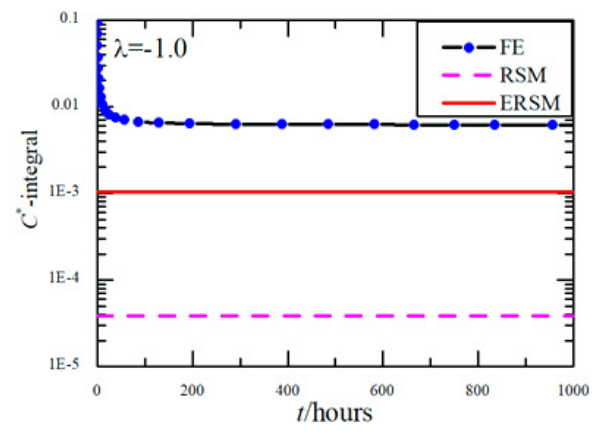

(b)

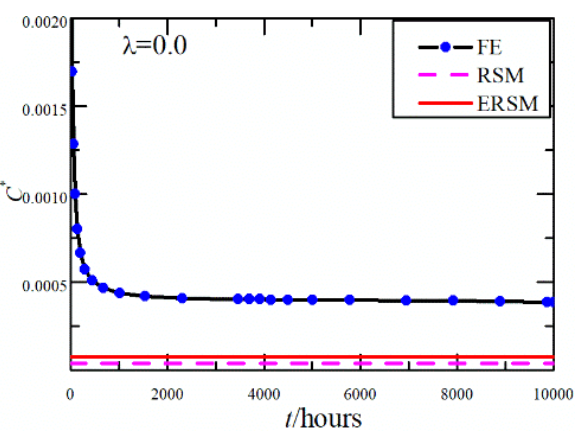

(d)

Fig. 3. Comparisons of $C^{*}$-integral obtained with RSM, ERSM and FE extraction for CCP with $a / W=0.15$ : (a) $\lambda=-1.5$; (b) $\lambda=-1.0$; (c) $\lambda=0.5$ and (d) $\lambda=0$.

The solutions of the $C^{*}$-integral computed with different methods for crack depths with $a / W=0.15$ and $a / W=0.5$ under different biaxial loading conditions are given in Figs. 3 and 4 respectively. It can be seen that the discrepancies between the results calculated with RSM and solutions computed by FE are obvious for shallow crack specimens, i.e., $a / W=0.15$. However, it can be found that $C^{*}$-integral under biaxial loading through ERSM agrees quite better with the solutions computed with ABAQUS than those computed with RSM. For deep crack specimens, the solutions predicted with ERSM proposed in this paper agree more closely with the FE solutions, however, the traditional solutions calculated with RSM deviates from the FE solutions greatly. In general, the solutions computed with ERSM are better than that of RSM.

Figure 5 is presented to better understand the variations of $C^{*}$-integral with different biaxial ratios. It can be seen that the solutions calculated with traditional RSM deviate from the FE solutions extracted from ABAQUS significantly. However, the results calculated by ERSM agree quite closely with those numerical computations. It implies the efficient and superiority of the proposed ERSM. Especially, the closer of solutions obtained by FE and RSM, the smaller of the biaxial ratio will be. 


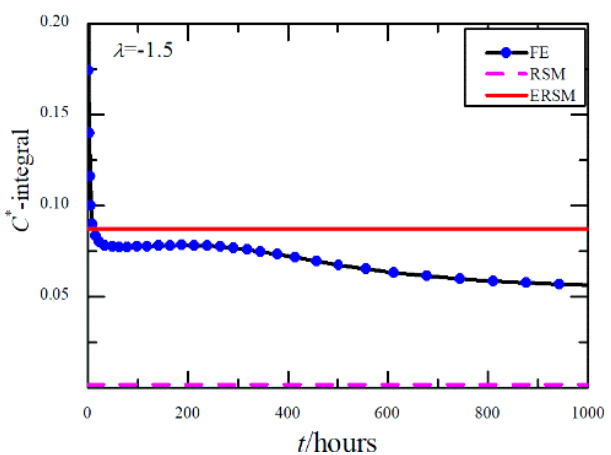

(a)

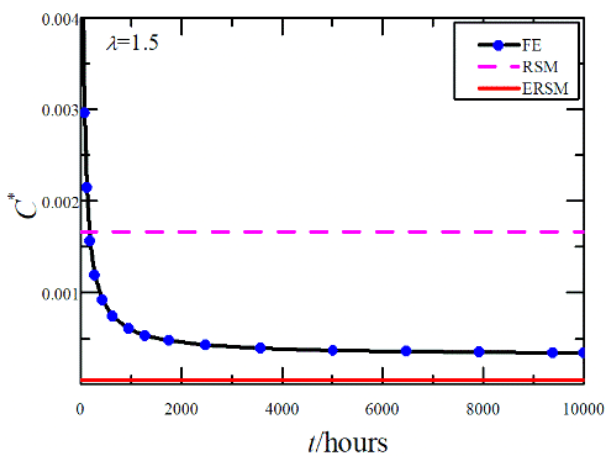

(c)

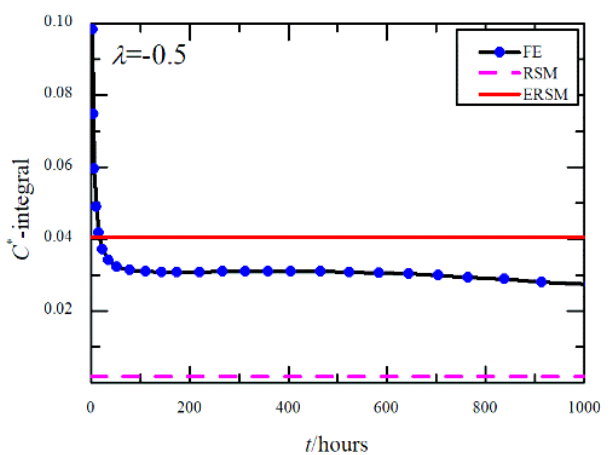

(b)

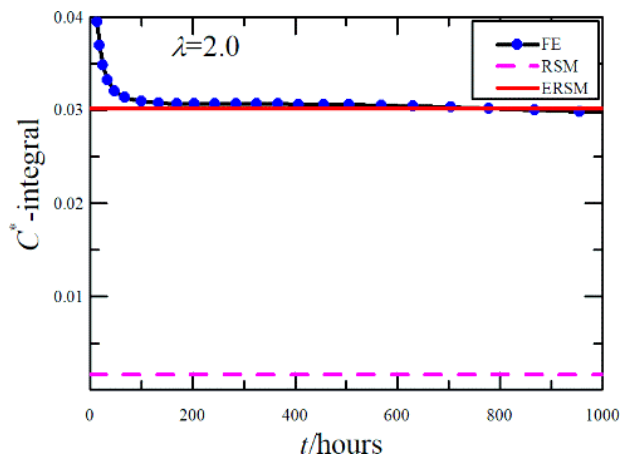

(d)

Fig. 4. Comparisons of $C^{*}$-integral obtained with RSM, ERSM and FE extraction for CCP with $a / W=0.50$ : (a) $\lambda=-1.5$; (b) $\lambda=-0.5$; (c) $\lambda=1.5$ and (d) $\lambda=2$.

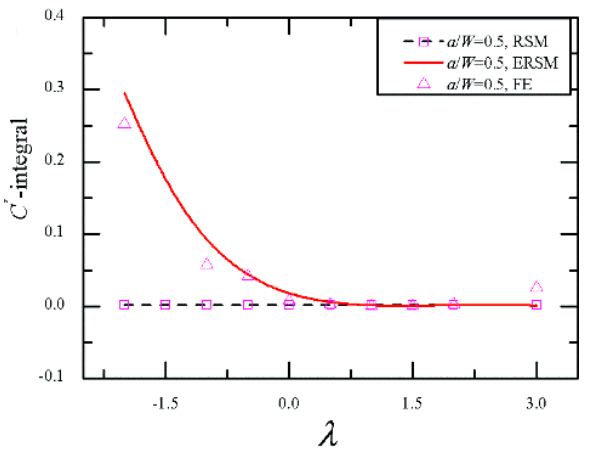

(a)

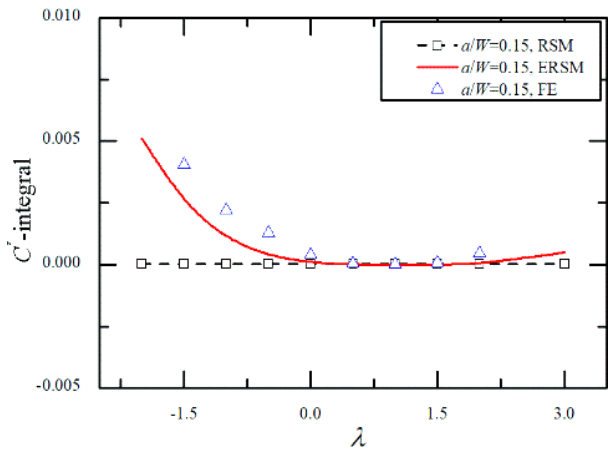

(b)

Fig. 5. Comparisons of $C^{*}$-integral obtained with RSM, ERSM and FE extractions under different biaxiality ratios for $\mathrm{CCP}$. 


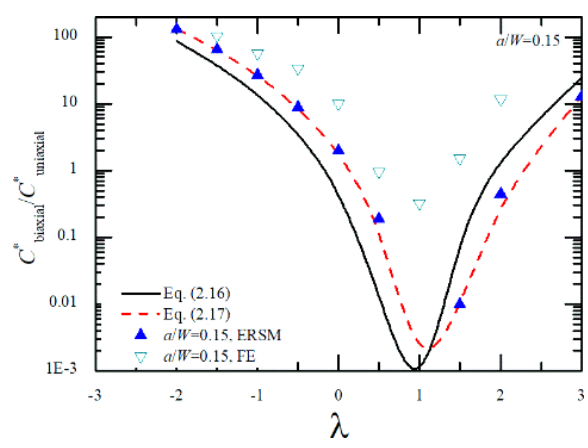

(a)

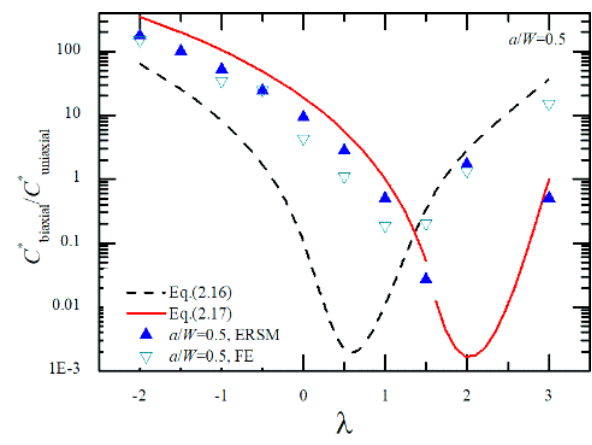

(b)

Fig. 6. Comparisons of $C_{\text {biaixial }}^{*} / C_{\text {uniaxial }}^{*}$ obtained with ERSM and FE solutions under different biaxiality ratios.

It can be found that the $C^{*}$-integral with negative stress biaxiality ratios is higher than that of $C^{*}$-integral under uniaxial and positive stress biaxiality ratios. Under the same loading, the value of $C^{*}$-integral with $a / W=0.5$ is greater than that of $a / W=0.15$. Furthermore, it can be seen that the solutions presented with ERSM agree with the solutions computed via FE very well. However, the solutions with traditional RSM are not as good as the ERSM presented in this paper.

The variations of ratio of $C^{*}$-integral under biaxial loading and uniaxial loading with biaxiality with different crack depths are given in Fig. 6. It is seen that the ratio of $C^{*}$-integral between biaxial loading and that of uniaxial loading varies differently. For shallow crack condition, the $C^{*}$-integral decreases firstly with the increase of biaxiality. $C^{*}$-integral approaches to the lowest value around biaxiality 1 . The solutions computed with ERSM agree with the numerical computations quite closely. For deep crack specimen, similar variation tendencies can be also found. The lowest $C^{*}$-integral is around biaxiality 1.5 . Those solutions present the " $V$ " shape variation tendencies. It also implies that the $C^{*}$-integral is highly dependent on the biaxial loading state.

\subsection{Relations of $C(t)$ and $C^{*}$-integral under biaxial loading}

There are many estimation formulae for the relationship between $C(t)$-integral and $C^{*}$-integral of power law creep constitutive equation. Riedel 2014] presented an approximation between $C(t)$-integral and $C^{*}$-integral as follows:

$$
\frac{C(t)}{C^{*}}=1+\frac{1}{n+1} \frac{t_{\mathrm{red}}}{t}
$$

Compared with Eq. (4.1), Ainsworth and Budden [1990] proposed another different relationship between $C(t)$-integral and $C^{*}$-integral as follows:

$$
\frac{C(t)}{C^{*}}=\frac{\left(1+t / t_{\mathrm{red}}\right)^{n+1}}{\left(1+t / t_{\mathrm{red}}\right)^{n+1}-1}
$$




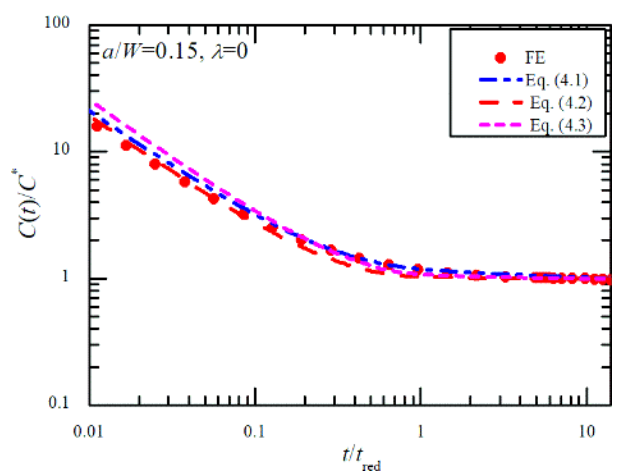

(a)

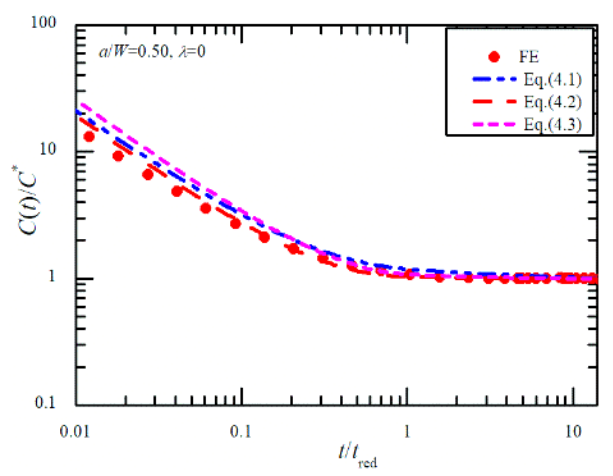

(b)

Fig. 7. Comparisons of $C(t) / C^{*}$ relationship in CCP under uniaxial loading for (a) $a / W=0.15$ and (b) $a / W=0.50$.

Similarly, Kim et al. 2001] presented another relationship as follows:

$$
\frac{C(t)}{C^{*}}=\frac{\left(1+t / t_{\mathrm{red}}\right)^{4.5}}{\left(1+t / t_{\mathrm{red}}\right)^{4.5}-1}
$$

It should be pointed out that the applicability of these relationships to biaxial loading conditions is still unknown. Hence, these relationships are discussed in what follows. Comparisons of $C(t)$ and $C^{*}$ relationships of those equations under uniaxial loading condition are given in Fig. 7 It can be seen that those proposed relations agree quite well in Fig. 7 regardless of crack depths.

The variation tendencies of $C(t) \sim C^{*}$ relationships for CCP with shallow crack and deep crack are given in Figs. 8 and 9 respectively. It can be deduced that the traditional formula of $C(t) \sim C^{*}$ relations are still applicable under biaxial loading conditions though the loading state is under biaxial condition.

\subsection{Discussions}

From the numerical computation and numerical analysis, it can be found that the $C^{*}$-integral is strongly dependent on its biaxiality. It varies with the variations of biaxiality. The reason is analyzed as follows.

For a biaxial loading state, the von Mises stress can be obtained as follows:

$$
2 \sigma_{y}^{2}=\sqrt{\left(\sigma_{1}-\sigma_{2}\right)^{2}+\left(\sigma_{2}-\sigma_{3}\right)^{2}+\left(\sigma_{1}-\sigma_{3}\right)^{2}}
$$

where $\sigma_{1}, \sigma_{2}$ and $\sigma_{3}$ are the principle stresses in three different directions and $\sigma_{y}$ is the yielding stress. For the two-dimensional condition, Eq. (4.4) is simplified as follows:

$$
\sigma_{y}^{2}=\sqrt{\sigma_{1}^{2}+\sigma_{2}^{2}-\sigma_{1} \sigma_{2}}
$$




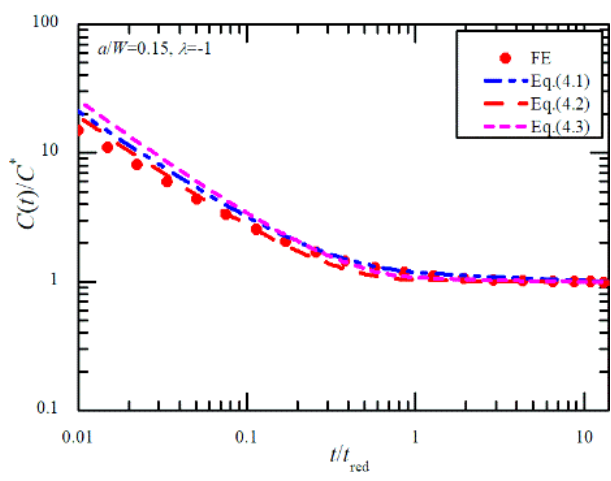

(a)

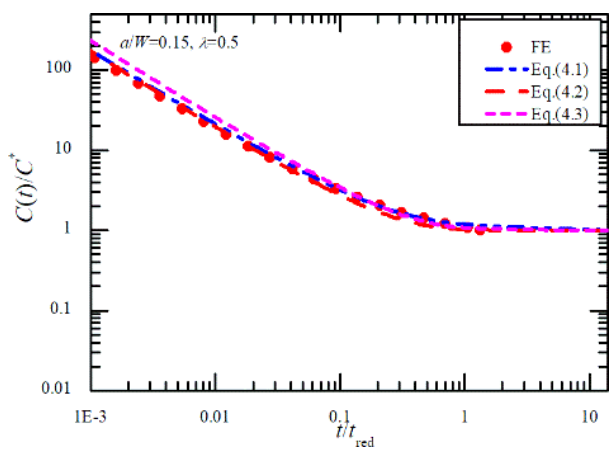

(c)

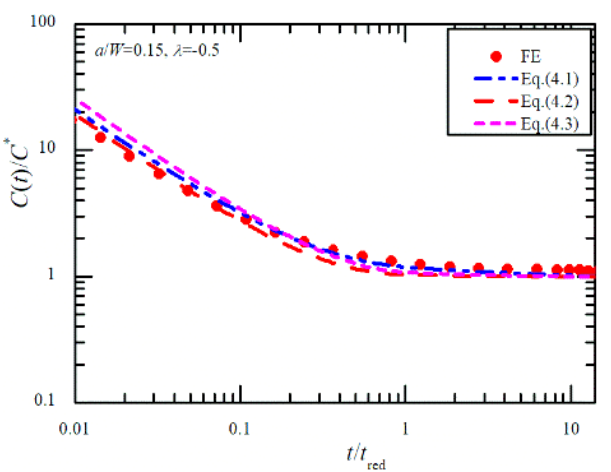

(b)

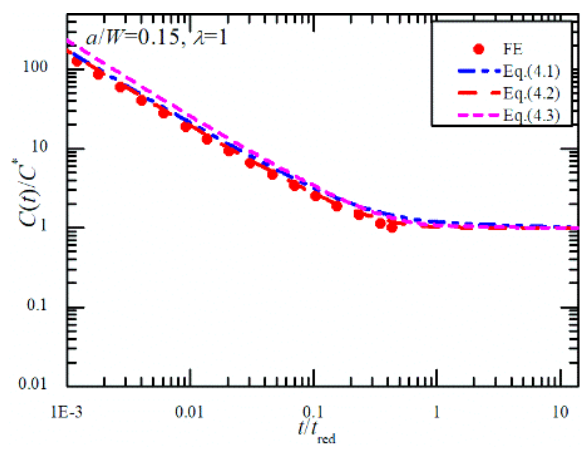

(d)

Fig. 8. Comparisons of $C(t) / C^{*}$ relationship for CCP under different biaxialities for $a / W=0.15$.

For CCP under biaxial loading, the equivalent stress is presented as follows:

$$
\sigma_{e q}=\sqrt{\lambda^{2}-\lambda+1 S_{y}}
$$

where $S_{y}$ is the loading in $y$-direction as shown in Fig. 1. From Eq. (4.6), it can be found that equivalent stress obtains the smallest value for $\lambda=0.5$. Hence, there is no difficulty to understand that the solution of $C^{*}$-integral is lowest under this condition.

The variations of $C(t) / C^{*}$ for $\mathrm{CCP}$ with $a / W=0.15$ under $\lambda=1.0$ are shown in Fig. 10. It should be known that variations of $C(t) \sim C^{* *}$ with different selections of $C^{*}$-integral where the $C^{*}$-integral here are all from the $C(t)$-integral with path-independence. Three different kinds of loading levels are obtained here, i.e., the curve I with $C^{*}=0.00536 \mathrm{~N} / \mathrm{mmh}$, curve II with $C^{*}=0.000346 \mathrm{~N} / \mathrm{mmh}$ and curve III with $C^{*}=3.751 \times 10^{-5} \mathrm{~N} / \mathrm{mmh}$ are very similar to each other, and the difference is the offsets between them. Obviously, the selection of $C^{*}$-integral for pathindependence solutions does not influence the shape and tendency of $C(t) \sim C^{*}$. 


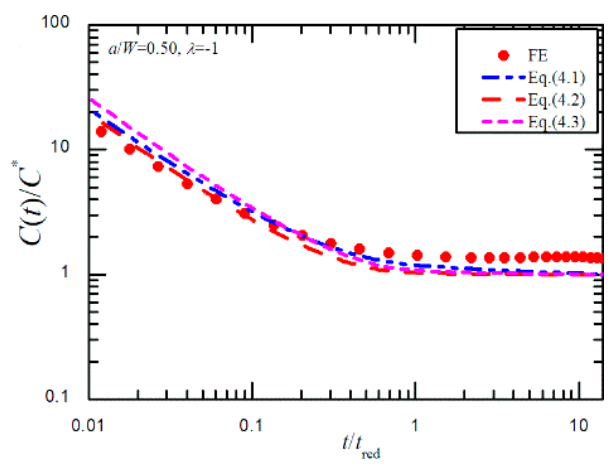

(a)

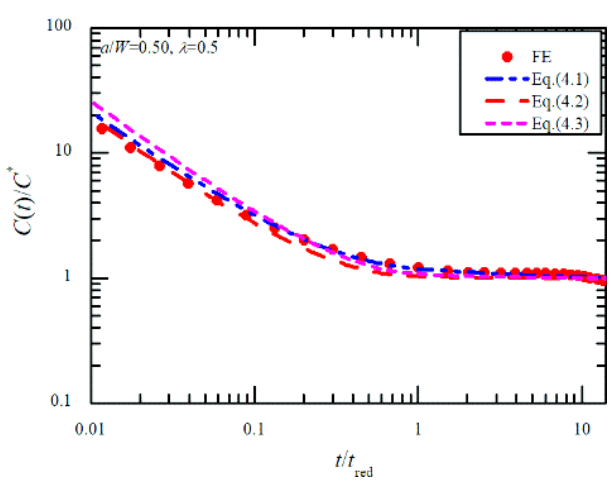

(c)

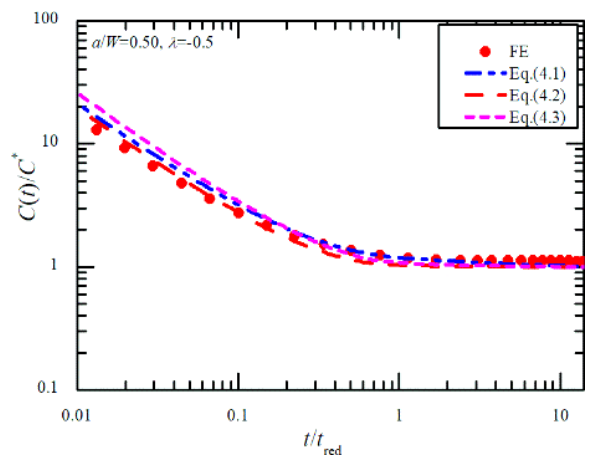

(b)

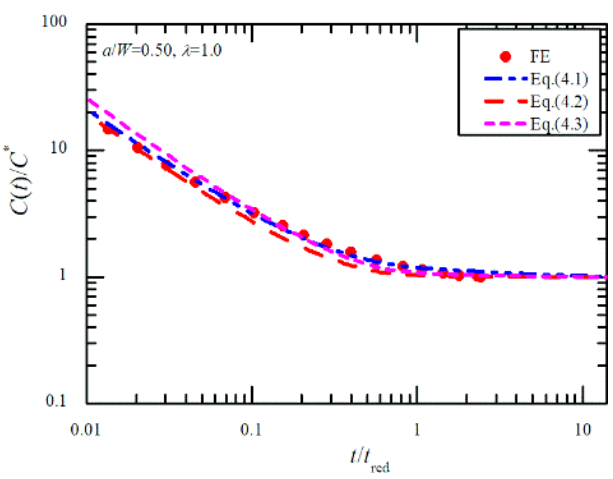

(d)

Fig. 9. $C(t) / C^{*}$ relationship for $\mathrm{CCP}$ under different biaxialities for $a / W=0.5$.

Hence, the $C^{*}$-integral with contour integral is not only path-independence, but also at the "platform" region. In engineering practices, the relationship here can be used to check out the accuracy of $C^{*}$-integral. For an accurate $C^{*}$-integral, $C(t) \sim C^{*}$ relationships are satisfied perfectly. If the $C(t) \sim C^{*}$ relationships are not satisfied, the $C^{*}$-integral computed may be not correct.

The effect of biaxiality on the $C^{*}$-integral is mainly caused by the increase of loading level. However, the variation tendencies between different biaxialities still obey the relations which are not specified with biaxial consideration (see Eqs. (4.1)(4.3) ). The main reason is that the $C^{*}$-integral is essence of a line integral which can reflect the input or output of deformation energy ahead of crack tip. Thus, those relations, which are derived with the crack tip characteristics, have incorporated the biaxial condition. However, RSM is a kind of simplified method to evaluate $C^{*}$ integral with rough accuracy where the estimation accuracy will be influenced by biaxial loading. Hence, the usage of RSM to estimate of $C^{*}$-integral under biaxial loading state should be very careful as the accuracy of this method is not that high. However, the proposed ERSM in this paper can help improve the estimation 


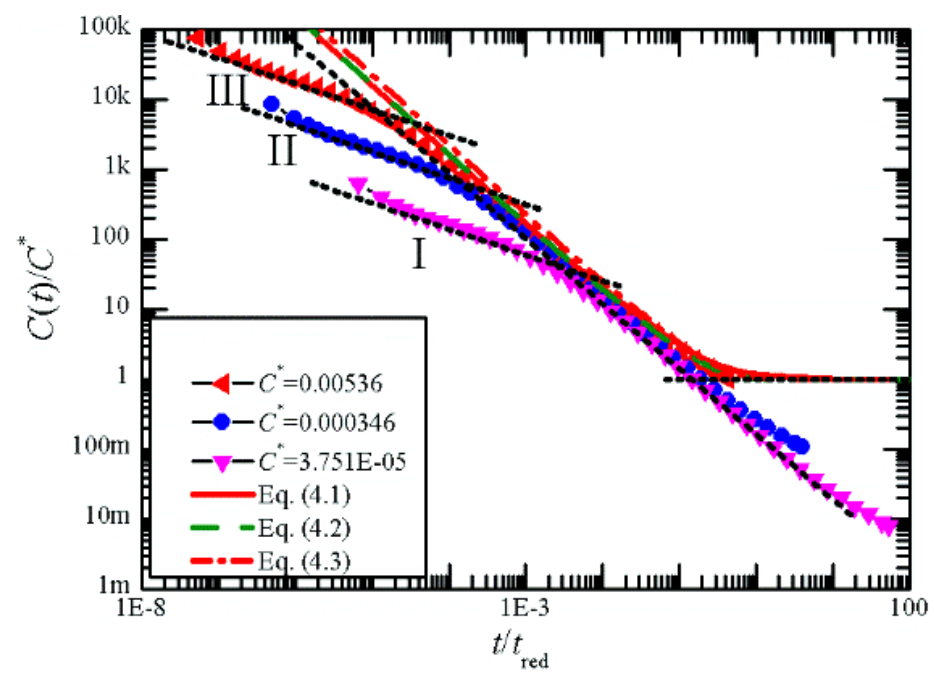

Fig. 10. Variations of $C(t) / C^{*}$ with different levels of $C^{*}$-integral.

accuracy. It has to be mentioned that ERSM and RSM are all engineering estimation methods, and there could be some errors between those engineering methods and accurate solutions. In general, ERSM presents a better estimation results compared with that of RSM. Although lots of biaxial ratios have been considered in this investigation, the biaxial ratio is always not allowed to differ significantly for the purpose of engineering safety in actual engineering practices. Hence, ERSM can provide reasonable solutions with a relative larger range of biaxial ratios.

\section{Concluding Remarks}

The estimation of $C^{*}$-integral under biaxial loading is investigated in this paper. Through theoretical analysis and numerical computations, the following conclusions are obtained:

(1) A theoretical formula is presented to estimate the ratio of $C^{*}$-integral under biaxial loading and uniaxial loading for CCP. The $C^{*}$-integral is highly dependent on the biaxial stress state, material properties of creep constants and crack depth for CCP. It is found that the increase of equivalent loading level heightens the level of $C^{*}$-integral. The variation tendencies for the ratio of $C^{*}$-integral between biaxial loading and uniaxial loading for CCP with biaxiality present to be " $V$ " shape.

(2) An ERSM considering biaxial effect is reported. The presented ERSM is verified with a good agreement with the numerical solution compared with that of traditional RSM. The ERSM proposed in this paper can be extended to the other conditions under biaxial loading for crack contained structural integral assessment considering creep behaviors. 
(3) The variation of $C(t) / C^{*}$ with normalized creep time is discussed in this paper. The classical relationship of $C(t) / C^{*}$ is still applicable under biaxial loading condition. The reason is that $C^{*}$-integral is a kind of line integral which is dependent on the energy state ahead of crack tip. Different biaxial ratios will lead to the variations of $C^{*}$-integral levels, however, they will not affect the slopes of $C(t) / C^{*}$ relations within different creep ranges for CCP.

\section{Acknowledgments}

The authors acknowledge the supports from the National Natural Science Foundation of China (11902009, 11672009 and 11672147), the Beijing Natural Science Foundation (2204074), the Scientific Research Common Program of Beijing Municipal Commission of Education (KM202010005034), the China Postdoctoral Science Foundation (2019M650403) and the Chaoyang District Postdoctoral Science Foundation (2019ZZ-47).

\section{References}

Ainsworth, R. A. [1984] "The assessment of defects in structures of strain hardening material," Engineering Fracture Mechanics 19, 633-642.

Ainsworth, R. A. and Budden, P. [1990] "Crack tip fields under non-steady steady state creep conditions-I. Estimates of the amplitude of the fields," Fatigue and Fracture of Engineering Materials and Structures 13, 263-276.

Ainsworth, R. A. and Budden, P. [1994] "Design and assessment of components subjected to creep," The Journal of Strain Analysis for Engineering Design 29, 201-207.

Assire, A., Michel, B. and Raous, M. [2001] "Creep crack initiation and creep crack growth assessments in welded structures," Nuclear Engineering and Design 206, $45-56$.

Dai, Y. W., Liu, Y. H., Qin, F., Qian, G. A. and Chao, Y. J. [2019a] " $C(t)$ dominance of the mixed I/II creep crack: Part I. Transient creep," Theoretical and Applied Fracture Mechanics 103, 102314.

Dai,Y. W., Liu, Y. H., Qin, F., Chao, Y. J. and Berto, F. [2019b] "Estimation of stress field for sharp V-notch in power-law creeping solids: An asymptotic viewpoint," International Journal of Solids and Structures 180-181, 189-204.

Dai, Y. W., Liu, Y. H., Qin, F., Chao, Y. J. and Chen, H. F. [2020a] "Constraint modified time dependent failure assessment diagram (TDFAD) based on $C(t)-A_{2}(t)$ theory for creep crack," International Journal of Mechanical Sciences 165, 105193.

Dai, Y. W., Liu, Y. H., Qin, F., Chao, Y. J. and Qian, G. A. [2020b] " $C(t)$ dominance of the mixed I/II creep crack: Part II. Extensive creep," Theoretical and Applied Fracture Mechanics 106, 102489.

Du, X. H., Zhang, J., Peng, H. and Liu, Y. H. [2017] "Plastic limit analysis of modified $9 \mathrm{Cr}-1 \mathrm{Mo}$ steel pressure vessel containing volume defect with creep damage law," International Journal of Applied Mechanics 9(2), 1750025.

Fujioka, T. and Ainsworth, R. A. [2013] "Simplified estimates of the creep crack growth parameter $\mathrm{C}(\mathrm{t})$ under primary/secondary stresses using the enhanced reference stress method," International Journal of Pressure Vessels and Piping 110, $32-41$. 
Kashkoli, M. D., Tahan, K. N. and Nejad, M. Z. [2018] "Thermomechanical creep analysis of FGM thick cylindrical pressure vessels with variable thickness," International Journal of Applied Mechanics 10(1), 1850008.

Kim, Y. J. [2001] "Contour integral calculations for generalised creep laws within abaqus," International Journal of Pressure Vessels and Piping 78, 661-666.

Kim, Y. J. and Budden, P. J. [2002] "Reference stress approximations for J and COD of circumferential through-wall cracked pipes," International Journal of Fracture 116(3), 195-218.

Kim, Y. J., Dean, D. and Budden, P. [2001] "Finite element analysis to assess the effect of initial plasticity on transient creep for defects under mechanical loading," International Journal of Pressure Vessels and Piping 78, 1021-1029.

Kim, Y. J., Huh, N. S. and Kim, Y. J. [2001] "Enhanced reference stress-based J and crack opening displacement estimation method for leak-before-break analysis and comparison with GE. EPRI method," Fatigue and Fracture of Engineering Materials and Structures 24, 243-254.

Kim, Y. J., Kim, J. S., Huh, N. S. and Kim, Y. J. [2002] "Engineering C-integral estimates for generalised creep behaviour and finite element validation," International Journal of Pressure Vessels and Piping 79, 427-443.

Kobayashi, H., Ohki, R., Itoh, T. and Sakane, M. [2017] "Multiaxial creep damage and lifetime evaluation under biaxial and triaxial stresses for type 304 stainless steel," Engineering Fracture Mechanics 174, 30-43.

Landes, J. and Begley, J. [1976] "A fracture mechanics approach to creep crack growth," Mechanics of Crack Growth (ASTM STP 590) ASTM, Philadelphia, PA, pp. 128148.

Lei, Y. [2004] " J-integral and limit load analysis of semi-elliptical surface cracks in plates under combined tension and bending," International Journal of Pressure Vessels and Piping 81, 43-56.

Lv, J., Yu, L., Du, W. and Li, Q. [2018] "Theoretical approach of characterizing the crack-tip constraint effects associated with material's fracture toughness," Archive of Applied Mechanics 88, 1637-1656.

Madia, M., Arafah, D. and Zerbst, U. [2014] "Reference load solutions for plates with semielliptical surface cracks subjected to biaxial tensile loading," International Journal of Pressure Vessels and Piping 119, 19-28.

Meek, C. and Ainsworth, R. A. [2015] "The effects of load biaxiality and plate length on the limit load of a centre-cracked plate," Engineering Fracture Mechanics 147, $306-17$.

Miura, N. and Takahashi, Y. [2010] "Evaluation of J-integral for surface cracked plates under biaxial loading using extended reference stress method," International Journal of Pressure Vessels and Piping 87, 58-65.

Oh, C. S., Kim, Y. J., Yoon, K. B., Ainsworth, R. A. and Nikbin, K. [2010] "The relationship between $\mathrm{Ct}$ and contour integrals under small-scale transient creep," Engineering Fracture Mechanics 77, 565-574.

Pan, S. X., Li, Q. and Liu, Q. D. [2017] "Ferroelectric creep associated with domain switching emission in the cracked ferroelectrics," Computational Materials Science 140, 244-252.

Riedel, H. [2014] Fracture at High Temperatures (Springer).

R6. [2010] Assessment of the Integrity of Structures Containing Defects, Revision 4.

Saxena, A. [1998] Nonlinear Fracture Mechanics for Engineers (CRC Press).

Xuan, F. Z., Si, J. and Tu, S. T. [2009] "Evaluation of $C^{*}$ integral for interacting cracks in plates under tension," Engineering Fracture Mechanics 76, 2192-2201. 
Y. Dai et al.

Xu, L. Y., Zhao, L., Jing, H. Y. and Han, Y. D. [2017] "Evaluation of multiple cracks interaction effect subjected to biaxial tension under creep regime," International Journal of Mechanical Sciences 122, 203-214.

Zhao, L., Xu, L., Han, Y. and Jing, H. [2015] "Two-parameter characterization of constraint effect induced by specimen size on creep crack growth," Engineering Fracture Mechanics 143, 121-137. 